\title{
Zinc oxide-palladium material an efficient solar-light driven photocatalyst for degradation of congo red
}

\author{
Karuppannan Rokesh ${ }^{1}$, Kulandaivel Jeganathan ${ }^{2}$, Kandasamy Jothivenkatachalam ${ }^{1, *}$ \\ ${ }^{1}$ Department of Chemistry, Anna University - BIT Campus, Tiruchirappalli-620024, \\ Tamil Nadu, India \\ ${ }^{2}$ Centre for Nanoscience and Nanotechnology, School of Physics, Bharathidasan University, \\ Tiruchirappalli-620024, Tamil Nadu, India \\ *jothivenkat@yahoo.com
}

PACS 81.07.-b

DOI 10.17586/2220-8054-2016-7-4-740-746

\begin{abstract}
Zinc oxide - palladium nanocomposite material $(\mathrm{ZnO}-\mathrm{Pd})_{N C M}$ was prepared and then characterized by UV-Vis spectroscopy, photoluminescence spectroscopy, X-ray diffraction analysis, energy dispersive X-ray analysis and field emission-scanning electron microscopy. The photocatalytic performance of $(\mathrm{ZnO}-\mathrm{Pd})_{N C M}$ was investigated by degradation of Congo red under solar light irradiation. Zinc oxide modified with palladium metal could greatly enhance its photocatalytic activity and effectively degraded by Congo red dye.
\end{abstract}

Keywords: zinc oxide, palladium, nanocomposite, photocatalyst, congo red, degradation.

Received: 11 February 2016

Revised: 23 April 2016

\section{Introduction}

Dyes from textile industries strongly pollute water resources and produce serious environmental problems. Photocatalysis is an efficient technique to remove the dyes in waste water [1]. Nanostructured semiconductors and their composite materials have been preferred as catalyst (photocatalyst) for the photocatalysis process [2,3]. Zinc oxide $(\mathrm{ZnO})$ is a wide band gap $(3.37 \mathrm{eV})$ semiconductor material; it is used as one of the efficient photocatalyst and photoelectrocatalyst [4-6]. This compound can be prepared by several techniques with collection of different morphologies and sizes. The nanostructured $\mathrm{ZnO}$ and $\mathrm{ZnO}-$ metal nanocomposite materials have been found huge attention in the field of photocatalysis [7-9], because of their high surface-volume ratio, stability and surface charge (SPR effect of metal nanoparticles). The metal support with $\mathrm{ZnO}$ to increase the photoexcited electrons-hole charge separation, transfer process and decreases the electron-hole recombination rate. Moreover, metal increases the adsorption of pollutant on catalyst surfaces afterwards improved its photocatalytic oxidation and reduction process $[10,11]$. Once the $\mathrm{ZnO}-\mathrm{Pd}$ nanocomposite material was prepared, it was characterized and its photocatalyic activity was investigated by degradation of Congo red dye under solar light.

\section{Materials and methods}

\subsection{Materials}

The materials such as zinc acetate dihydrate, palladium acetate, hydrazine hydrate and were obtained from Merck, and sodium borohydride, congo red were purchased from S. D. Chemicals, Mumbai.

\subsection{Preparation of $\mathrm{ZnO}-\mathrm{Pd}$ nanocomposite material}

$\mathrm{ZnO}$ nanomaterial was prepared by simple microwave assisted technique. A $100 \mathrm{ml}$ mixture of $0.05 \mathrm{M}$ aq. zinc acetate and $0.2 \mathrm{M}$ aq. Hydrazine hydrate was stirred for $30 \mathrm{~min}$ then subjected to microwave irradiation $15 \mathrm{~min}$. The obtained white precipitate was washed, centrifuged and calcined at $200{ }^{\circ} \mathrm{C}$ for $2 \mathrm{hrs}$. Then, the prepared $\mathrm{ZnO}$ and palladium acetate precursor was added to an aqueous solution with 100:2.5 weight percentage and stirred for few hrs. Then, freshly prepared sodium borohydride solution was added to afford ZnO-Pd nanocomposite material.

\subsection{Characterization}

The prepared $\mathrm{ZnO}$ and $(\mathrm{ZnO}-\mathrm{Pd})_{N C M}$ materials absorbance spectra were recorded using a Shimadzu UV-2550 UV-Vis spectrometer. Photoluminescence was recorded using JASCO-FP-6500 spectrofluorometer. The crystalline nature of prepared materials was studied by XRD pattern using a Bruker Advance with $\mathrm{Cu} \mathrm{K} \alpha$ radiation. The morphology and elemental composition of the samples were recorded by Carl Zeiss FE-SEM and Bruker EDX instruments. 


\subsection{Photocatalytic study}

The photocatalytic experiment was carried out by photodegradation of Congo red (CR) under solar light irradiation. A $20 \mathrm{mg}$ of prepared $\mathrm{ZnO}$ and $\mathrm{ZnO}-\mathrm{Pd}$ composites material was suspended into $50 \mathrm{ml}$ of Congo red (CR) $25 \mathrm{ppm}$ aq. solution. Then the dye solution was exposed to solar light irradiation in open air condition, time between 11 am to $2 \mathrm{pm}$ with continuous aeration. Subsequently, at a given time interval 3 ml aliquots were collected and centrifuged. The degradation process was monitored and confirmed by UV-Vis absorbance spectroscopy by measuring its absorption.

\section{Results and discussion}

\subsection{Optical property}

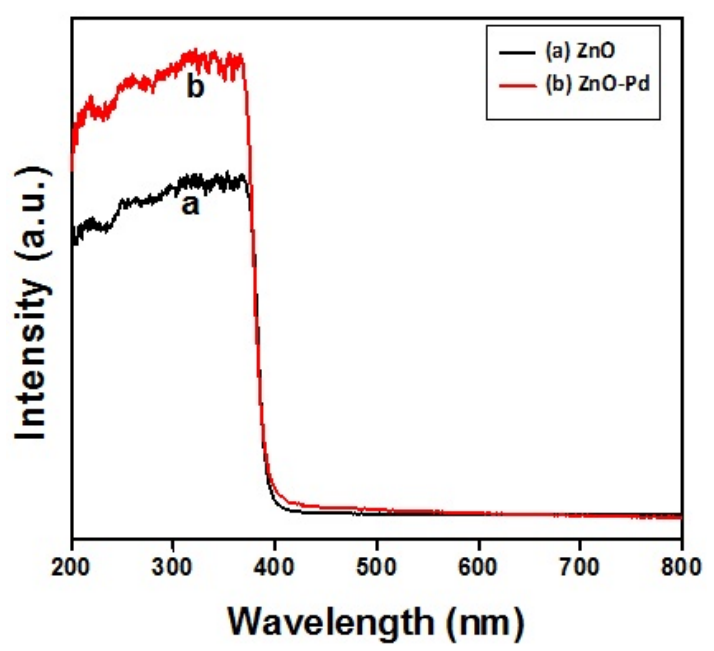

FIG. 1. UV-Diffuse reflectance spectra of (a) $\mathrm{ZnO}$ (b) $(\mathrm{ZnO}-\mathrm{Pd})_{N C M}$

The UV-DRS measurement reveals that the band energy and optical properties of semiconductors materials. UV-DRS spectra of the $\mathrm{ZnO}$ and $(\mathrm{ZnO}-\mathrm{Pd})_{N C M}$ samples were measured at room temperature and are shown in Fig. 1. The prepared $\mathrm{ZnO}$ and $(\mathrm{ZnO}-\mathrm{Pd})_{N C M}$ showed strong absorption below $400 \mathrm{~nm}$ in near-UV spectral region, confirming the wide band of $\mathrm{ZnO}$ semiconductor material. The $(\mathrm{ZnO}-\mathrm{Pd})_{N C M}$ does not show any absorption in the visible region due the particle size and amount of $\mathrm{Pd}$ in the composite is much less. Moreover, the $(\mathrm{ZnO}-\mathrm{Pd})_{N C M}$ have existed higher absorbance intensity than bare $\mathrm{ZnO}$ in UV region, which indicates that the Pd improves the quantum efficiency of the $\mathrm{ZnO}$ material [12].

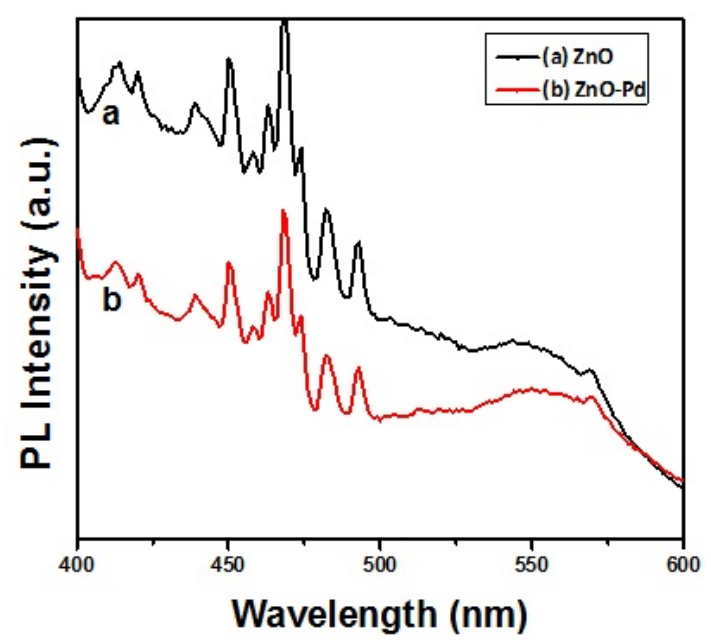

FIG. 2. Photoluminescence spectra (a) $\mathrm{ZnO}$ (b) $(\mathrm{ZnO}-\mathrm{Pd})_{N C M}$ 
The PL spectra showed wide band emission at visible region (Fig. 2). This extended emission is due to the recombination of photogenerated electron-hole and oxygen vacancy sites in the materials. The $\mathrm{ZnO}$ and ( $\mathrm{ZnO}-$ $\mathrm{Pd})_{N C M}$ where observed weak emission at $468 \mathrm{~nm}$ due to band edge free excitons. The blue and green emission peaks were observed around 520-580 nm for the prepared samples due to presence of oxygen vacancies in the material's surface [13]. The emission intensity of $\mathrm{ZnO}$ decreased with the addition of palladium metal because of the Pd trapped excited electrons from the zinc oxide.

\subsection{Crystalline properties}

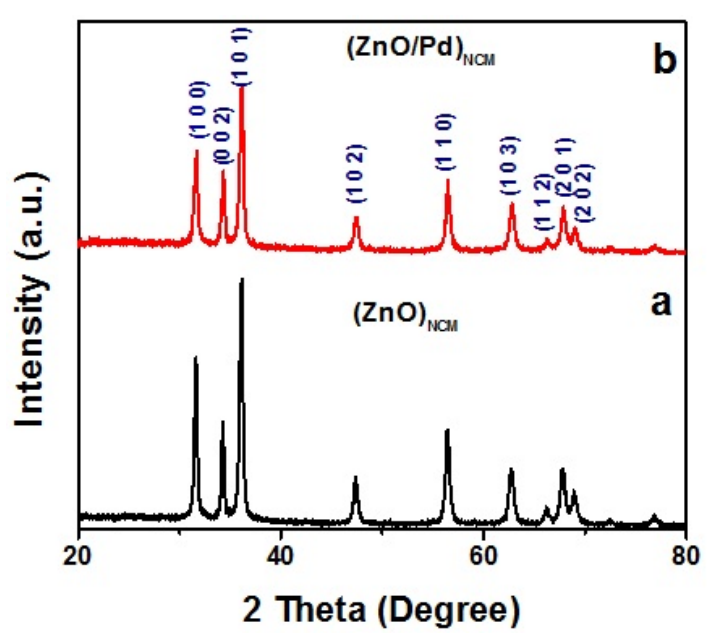

FIG. 3. XRD pattern of (a) $\mathrm{ZnO}$ and (b) $(\mathrm{ZnO}-\mathrm{Pd})_{N C M}$

The XRD pattern of $\mathrm{ZnO}$ and $(\mathrm{ZnO}-\mathrm{Pd})_{N C M}$ composite material is shown in Fig. 3. All the diffraction peaks in XRD are confirmed that the prepared $\mathrm{ZnO}$ in hexagonal wurtzite structure (JCPDS No. 36-1451). The diffraction peaks of palladium was not observed in $(\mathrm{ZnO}-\mathrm{Pd})_{N C M}$ due to the very minimum percentage of Pd present in the composite $[13,14]$. The absence of any other peak in XRD is confirmed that the prepared materials are highly pure.

\subsection{Morphology and elemental analysis}

The morphology and elemental composition of synthesized materials were studied by FE-SEM and EDX analysis is shown in Fig. 4. The synthesized $\mathrm{ZnO}$ and $\mathrm{ZnO}-\mathrm{Pd}$ composite material displays flower buds-like morphology with average size about 1 to $2 \mu \mathrm{m}$ (Fig. 4(a,c)). The spherical like Pd nanoparticles are deposited on $\mathrm{ZnO}$ surface can be confirmed by FE-SEM image. The $\mathrm{ZnO}$ buds have exhibited rough surface which formed by the agglomeration of small nanoparticles. EDX analysis provided the elemental composition of the materials is shown in Fig. 4(b,d). In Fig. 4(d) the spectrum confirms that the $\mathrm{Zn}, \mathrm{O}$ and $\mathrm{Pd}$ elements are present in the composite material, as evidence for formation of $(\mathrm{ZnO}-\mathrm{Pd})_{N C M}$. The absence of any other elemental peaks in EDX spectrum confirmed that the as-synthesized materials were very pure.

\subsection{Photocatalytic study}

The photocatalytic experiment was carried out by photodegradation of Congo red (CR) under solar light irradiation. The effect of different parameters such as catalyst loading, solution $\mathrm{pH}$ and dye concentration on photodegradation efficiency were evaluated using $\mathrm{ZnO}$ and $(\mathrm{ZnO}-\mathrm{Pd})_{N C M}[15,16]$.

The experiment was carried out by $25 \mathrm{ppm}(\mathrm{mg} / \mathrm{l})$ of CR in aqueous solution in the presence of $20 \mathrm{mg} / 50 \mathrm{ml}$ of $\mathrm{ZnO}$ and $(\mathrm{ZnO}-\mathrm{Pd})_{N C M}$ photocatalysts. The percentage of degradation was significantly increased for the zinc oxide - palladium nanocomposite and nearly attained maximum degradation efficiency of $100 \%$ in 3 hrs under solar irradiation. The result displays that the photocatalytic activities of $(\mathrm{ZnO}-\mathrm{Pd})_{N C M}$ are higher than that of $\mathrm{ZnO}$, shown in Fig. 5. Because the palladium metal acts as electron sink, it therefore enhanced the electron-hole charge separation and reduced the charge recombination process. This process promotes the hydroxide radical and superoxide radical generation $[12,13,16]$. These generated active radicals are the effective degradation agents for the CR dye molecules. 

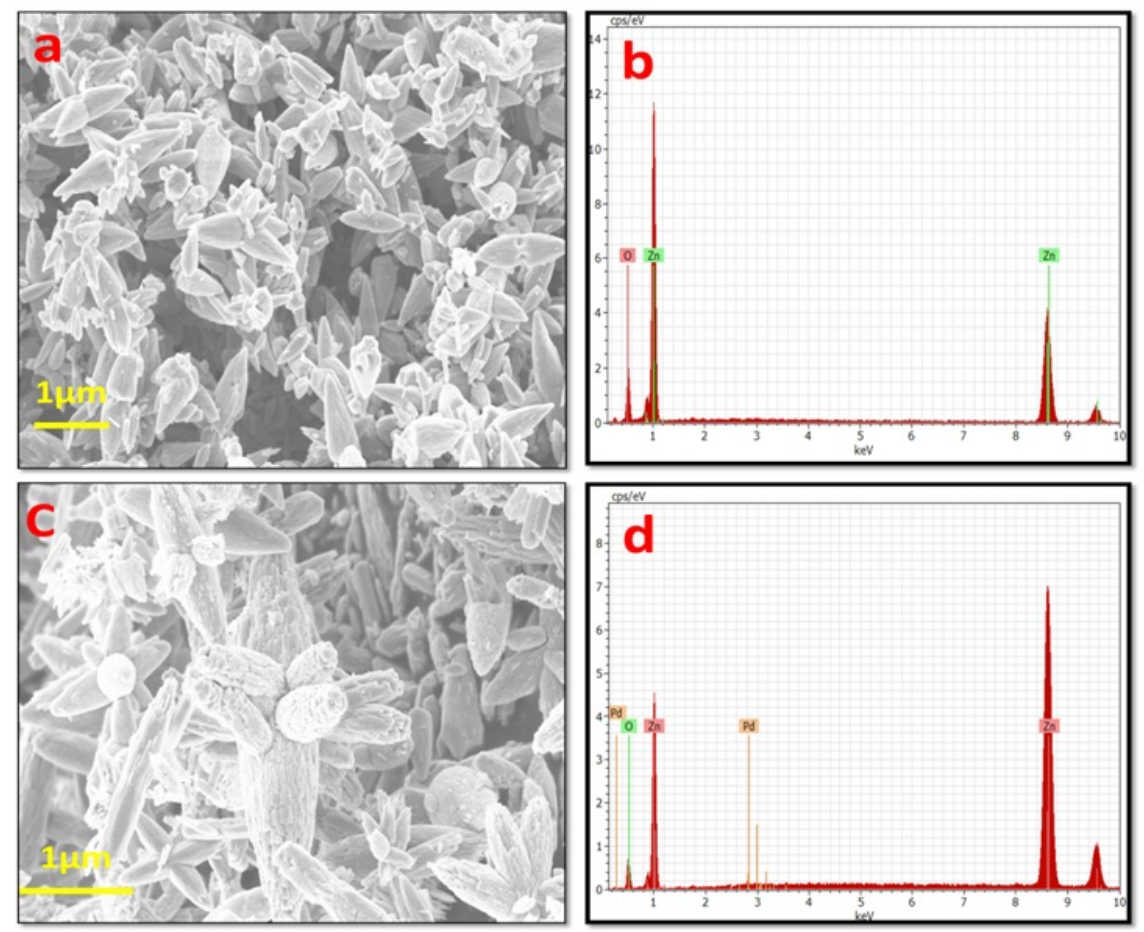

FIG. 4. FE-SEM and EDX spectrum of $\mathrm{ZnO}(\mathrm{a}, \mathrm{b})$ and $(\mathrm{ZnO}-\mathrm{Pd})_{N C M}(\mathrm{c}, \mathrm{d})$

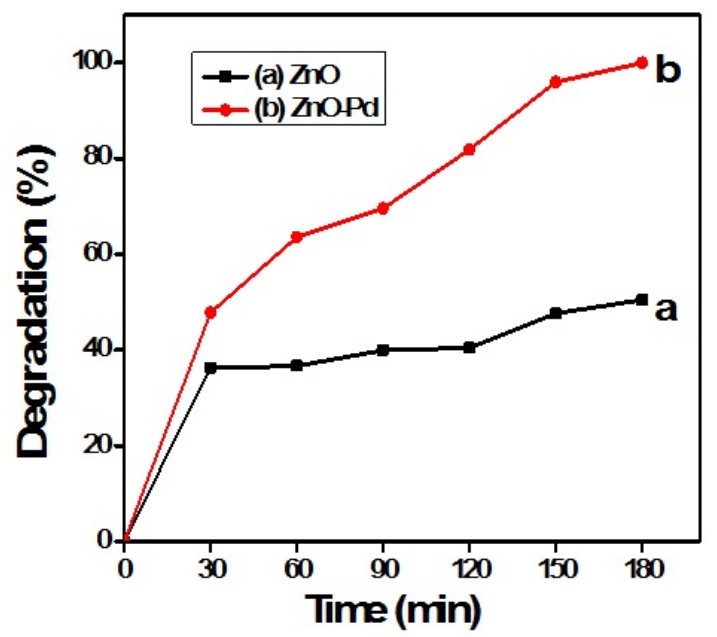

FIG. 5. The photocatalytic degradation of congo red (25 ppm) using (a) $\mathrm{ZnO}$ and ( $\mathrm{ZnO}-\mathrm{Pd})_{N C M}$ using $20 \mathrm{mg} / 50 \mathrm{ml}$

3.4.1. Effect of the catalyst loading. This experiment was carried out using different amounts of $(\mathrm{ZnO}-\mathrm{Pd})_{N C M}$ ( 5 to $40 \mathrm{mg} / 50 \mathrm{ml}$ ) catalyst and dye concentration $25 \mathrm{ppm}$. In Fig. 6, the results showed that the photodegradation efficiency was increased with increasing amounts of catalyst and the maximum efficiency was observed at 20$40 \mathrm{mg}$ of catalyst loading In this case, $20 \mathrm{mg} / 50 \mathrm{ml}$ catalyst amount was found to have the highest photodegradation efficiency with lower catalyst loading and it's also consider as the optimum amount of catalyst. In general, the amount of catalyst is proportional to the generation of number of active radical such as $\mathrm{OH}$ and $\mathrm{O} 2^{-}$which rapidly degrade the adsorbed dye molecules. A catalyst at higher loading increases the total active sites of the catalyst but dye molecules is constant at fixed concentration. Hence, the optimum amount of catalyst is enough for the efficient degradation of CR at above optimum concentration [17, 18].

3.4.2. Influence of $\mathrm{pH}$. The influence of initial dye $\mathrm{pH}$ value on the degradation efficiency of $\mathrm{CR}$ was investigated is shown in Fig. 7. The $\mathrm{pH}$ of dye solution was adjusted using dilute sulfuric acid or dilute sodium hydroxide. 


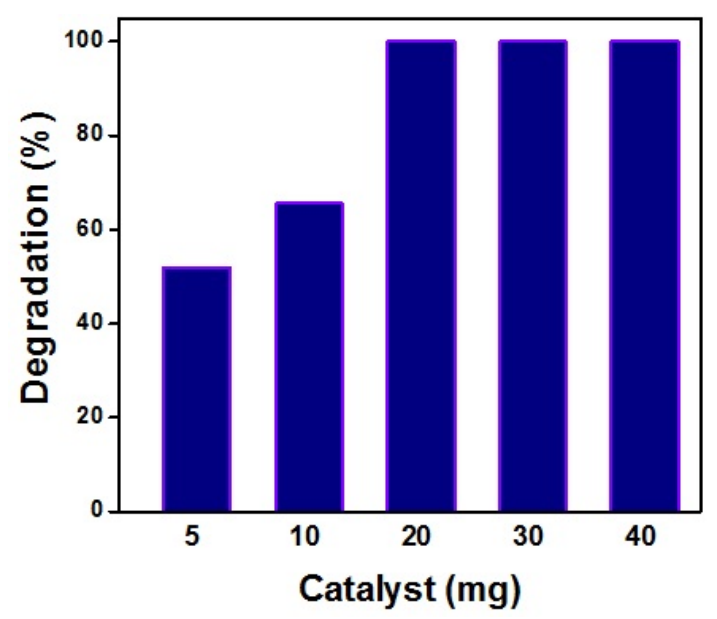

FIG. 6. Photocatalytic degradation of of congo red $(25 \mathrm{ppm})$ using $(\mathrm{ZnO}-\mathrm{Pd})_{N C M}$ with different amount of catalyst

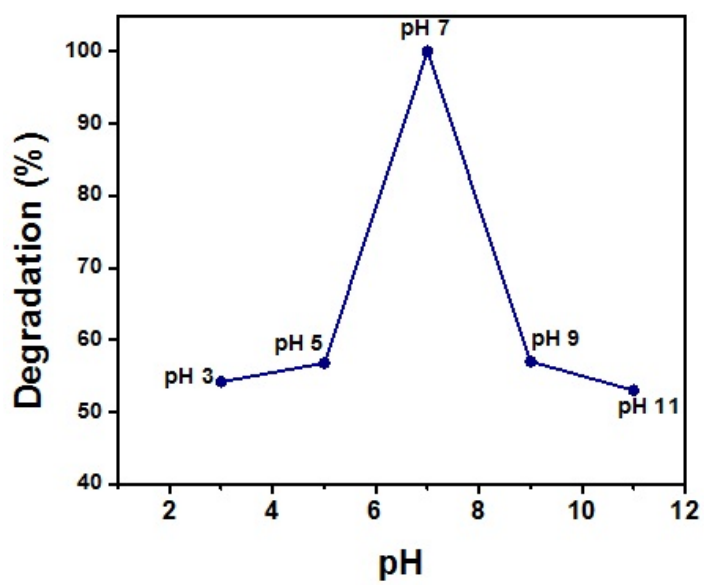

FIG. 7. Effect of dye solution $\mathrm{pH}$ on the photocatalytic degradation of congo red (25 ppm) using $(\mathrm{ZnO}-\mathrm{Pd})_{N C M} 20 \mathrm{mg} / 50 \mathrm{ml}$

The result showed that the higher degradation efficiency was observed at neutral $\mathrm{pH}(\mathrm{pH}$ 7). On the other hand, the degradation efficiency decreased at both acidic and basic $\mathrm{pH}$. From that result the photocatalytic degradation of $\mathrm{CR}$ is favorable at neutral $\mathrm{pH}$ in presence of $(\mathrm{ZnO}-\mathrm{Pd})_{N C M}$ [17-19].

3.4.3. Effect of the initial dye concentration. The effect of initial concentration of CR dye on photodegradation activity was studied by varying the CR dye concentration from to 10 to $40 \mathrm{ppm}$ (Fig. 8). The degradation efficiency of Congo red was high with increased dye concentration up to $25 \mathrm{ppm}$, however, after that, the degradation efficiency was decreased. This may be due to competition of adsorption between dye molecules and catalyst surface. At higher dye concentrations, the number of dye molecules is high, but the available active sites on the catalyst surface are constant, hence the fixed catalyst amount occupies a fixed number of active sites. Hence, it is possible to have a constant amount of dye molecule adsorption and degradation and it is not enough to degrade a large number of dye molecules (higher concentration) [16-19].

3.4.4. Photocatalytic mechanism. Photocatalytic mechanism of $\mathrm{ZnO}-\mathrm{Pd}$ nanocomposite for degradation of Congo red is shown in Fig. 9. When sunlight is exposed to the $\mathrm{ZnO}-\mathrm{Pd}$ nanocomposite, it undergoes photoexcitation followed by electron movement to the conduction band (CB) and the holes are generated in the valence band (VB) of $\mathrm{ZnO}$. The photoinduced holes are captured by surrounding water molecules, producing hydroxyl radicals $(\mathrm{OH})$. Then, the photoinduced electrons can be trapped by oxygen molecules on the catalyst surface and formed superoxide radicals $\left(\mathrm{O}_{2}^{-}\right)$. Afterward, the generated free radicals effectively degraded the $\mathrm{CR}$ dye molecules in aqueous solution. Moreover, the electron-hole can be recombining together during the light irradiation and this process is effectively prevented by palladium metal on zinc oxide's surface. At the time, the metal nanoparticle 


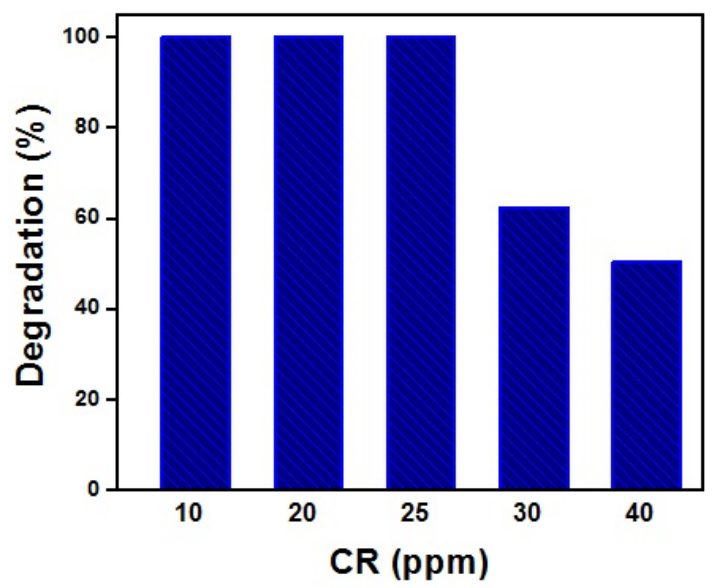

FIG. 8. Effect of initial congo red concentration on the photocatalytic degradation of congo red using $(\mathrm{ZnO}-\mathrm{Pd})_{N C M} 20 \mathrm{mg} / 50 \mathrm{ml}$
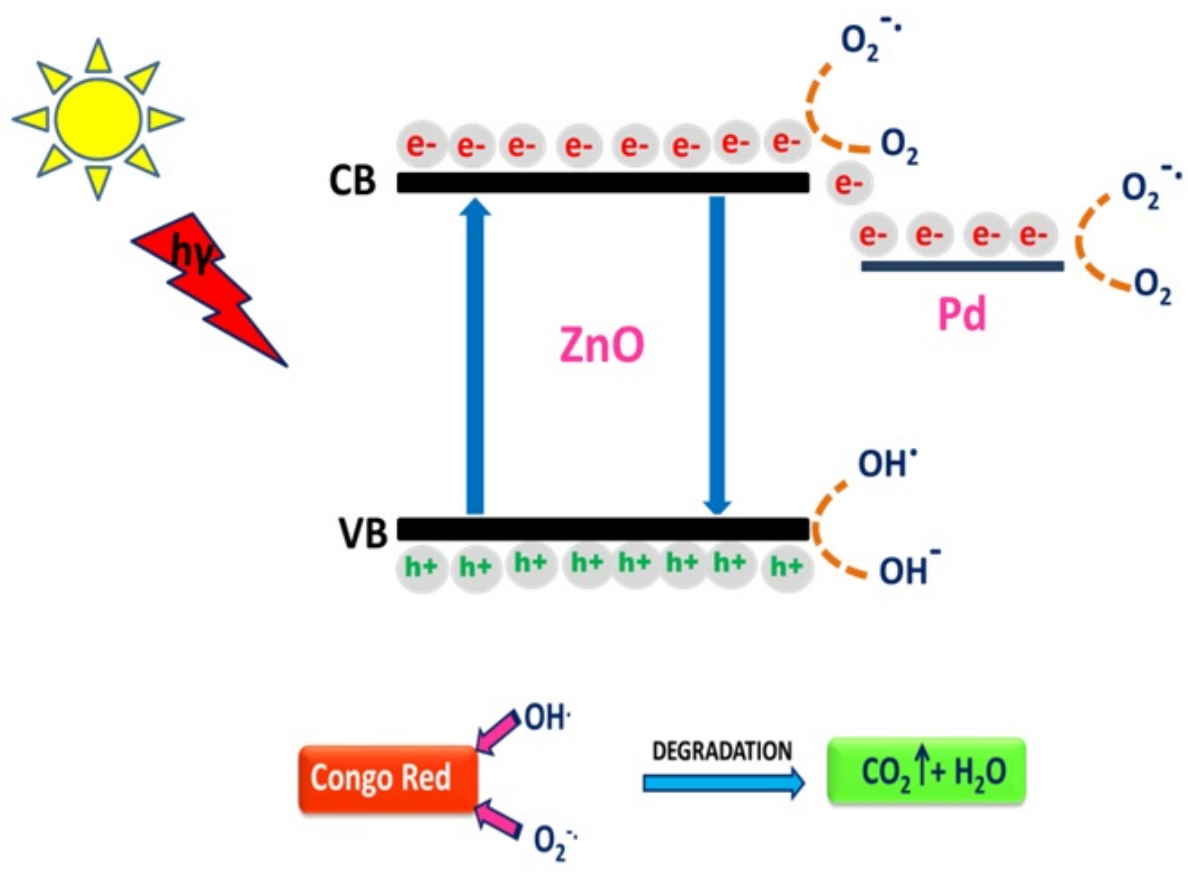

FIG. 9. Photocatalytic mechanism for ZnO-Pd nanocomposite-catalyzed degradation of Congo red

traps the surface electron from conduction band of $\mathrm{ZnO}$ and transfers it to oxygen molecules adsorbed on the catalyst surface $[12,13,20]$.

\section{Conclusion}

$\mathrm{ZnO}$ and $\mathrm{ZnO}-\mathrm{Pd}$ nanocomposite materials have been successfully synthesized and characterized by UV-DRS, PL, XRD, EDX and FE-SEM. The UV-DRS displayed the optical properties and PL spectra explained the photochemical charge separation and transfer properties of the materials. The XRD pattern confirmed crystalline nature and EDX analysis confirmed the elemental composition of the materials. The FE-SEM showed the surface morphology of $\mathrm{ZnO}$ and $\mathrm{ZnO}-\mathrm{Pd}$ nanocomposite materials. The photocatalytic activity of $(\mathrm{ZnO}-\mathrm{Pd})_{N C M}$ was investigated by the decomposition of Congo red under solar light irradiation. The zinc oxide-palladium nanocomposite material exhibited efficient photocatalytic activity due to synergic effect of palladium and promote the photocatalytic degradation of Congo red. 


\section{References}

[1] Paola A.D., Lopez E.G., Marci G., Palmisano L. A survey of photocatalytic materials for environmental remediation. J. Hazard. Mater, 2012, 3-29, P. 211-212.

[2] Hu X., Li G., Yu J.C. Design, fabrication, and modification of nanostructured semiconductor materials for environmental and energy application. Langmuir, 2010, 26(5), P. 3031-3039.

[3] Tong H., Ouyang S., Bi Y., Umezawa N., Oshikiri M., Ye J. Nano-photocatalytic materials: possibilities and challenges. Adv. Mater, 2012, 24, P. 229-251.

[4] Ahmad M., Zhu J. ZnO based advanced functional nanostructures: synthesis, properties and applications. J. Mater. Chem., 2011, 21, P. 599-614.

[5] Nithya A., Jothivenkatachalam K. Chitosan assisted synthesis of ZnO nanoparticles: an efficient solar light driven photocatalyst and evaluation of antibacterial activity. J. Mater. Sci.-Mater. Electron., 2015, 26(12), P. 10207-10216.

[6] Rokesh K., Pandikumar A., Chandra Mohan S., Jothivenkatachalam K. Aminosilicate sol-gel supported zinc oxide-silver nanocomposite material for photoelectrocatalytic oxidation of methanol. J. Alloys Compd., 201; DOI: 10.1016/j.jallcom.2016.04.089.

[7] Ansari S.A., Khan M.M., Ansari M.O., Lee J., Cho M.H. Biogenic synthesis, photocatalytic, and photoelectrochemical performance of Ag-ZnO nanocomposite. J. Phys. Chem. C, 2013, 117(51), P. 27023-27030.

[8] Li P., Wei Z., Wu T., Peng Q., Li Y. Au-ZnO hybrid nanopyramids and their photocatalytic properties. J. Am. Chem. Soc., 2011, 133, P. 5660-5663.

[9] Zong Y., Li Z., Wang X., Ma J., Men Y. Synthesis and high photocatalytic activity of Eu-doped ZnO nanoparticles. Ceram. Int., 2014, 40, P. 10375-10382.

[10] Xie W., Li Y., Sun W., Huang J., Xie H., Zhao X. Surface modification of ZnO with Ag improves its photocatalytic efficiency and photostability. J. Photochem. Photobiol. A, 2010, 216, P. 149-155.

[11] Georgekutty R., Seery M.K., Pillai S.C. A highly efficient Ag-ZnO photocatalyst: synthesis, properties, and mechanism. J. Phys. Chem. $C, 2008,112$, P. 13563-13570.

[12] Chang Y., Xu J., Zhang Y., Ma S., Xin L., Zhu L., Xu C. Optical properties and photocatalytic performances of Pd modified ZnO samples. J. Phys. Chem. C, 2009, 113, P. 18761-18767.

[13] Zhang Y., Wang Q., Xu J., Ma S. Synthesis of Pd/ZnO nanocomposites with high photocatalytic performance by a solvothermal method. Appl. Surf. Sci., 2012, 258(24), P. 10104-10109.

[14] Zeng Y., Lou Z., Wang L., Zou B., Zhang T., Zheng W., Zou G. Enhanced ammonia sensing performances of Pd-sensitized flowerlike ZnO nanostructure. Sens. Actuators, B, 2011, 156, P. 395-400.

[15] Zhong J.B., Li J.Z., He X.Y., Zeng J., Lu Y., Hu W., Lin K. Improved photocatalytic performance of Pd-doped ZnO. Curr. Appl. Phys., 2012, 12, P. 998-1001.

[16] Erdemoglu S., Aksu S.K., Sayılkan F., Izgi B., Asilturk M., Sayılkan H., Frimmel F., Guçer S. Photocatalytic degradation of congo red by hydrothermally synthesized nanocrystalline $\mathrm{TiO}_{2}$ and identification of degradation products by LC-MS. J. Hazard. Mater, 2008, 155, P. 469-476.

[17] Jothivenkatachalam K., Prabhu S., Chandra Mohan S., Jeganathan K. Solar, visible and UV light photocatalytic activity of CoWO 4 for the decolourization of methyl orange. Desalin. Water Treat., 2014, P. 1-12, DOI: 10.1080/19443994.2014.906324.

[18] Jothivenkatachalam K., Prabhu S., Nithya A., Jeganathan K. Facile synthesis of $\mathrm{WO}_{3}$ with reduced particle size on zeolite-Y and enhanced photocatalytic activity. RSC Adv, 2014, 4, P. 21221-21229.

[19] Subash B., Krishnakumar B., Swaminathan M., Shanthi M. Highly active Zr co-doped Ag-ZnO photocatalyst for the mineralization of acid black 1 under UV-A light illumination. Mater. Chem. Phys., 2013, 141, P. 114-120.

[20] Khalil A., Gondal M.A., Dastageer M.A. Augmented photocatalytic activity of palladium incorporated ZnO nanoparticles in the disinfection of Escherichia coli microorganism from water. Appl. Catal. A, 2011, 402, P. 162-167. 